\title{
TIC et nouveaux savoirs
}

ICT and new skills

TIC y nuevos conocimientos de enseñanza

\section{Georges-Louis Baron}

URL : http://journals.openedition.org/ries/2552

DOI : $10.4000 /$ ries. 2552

ISSN : 2261-4265

Éditeur

Centre international d'études pédagogiques

Édition imprimée

Date de publication : 1 mars 2000

Pagination : 13-18

ISSN : 1254-4590

Référence électronique

Georges-Louis Baron, "TIC et nouveaux savoirs », Revue internationale d'éducation de Sèvres [En ligne], 25 | 2000, mis en ligne le 01 mars 2003, consulté le 03 mai 2019. URL : http:// journals.openedition.org/ries/2552; DOI : 10.4000/ries.2552 


\section{TIC et nouveaux savoirs}

Georges-Louis Baron

Directeur du département technologies nouvelles et éducation de l'Institut national de recherche pédagogique, professeur en sciences de l'éducation

Il est banal de remarquer que les technologies actuelles offrent de nouvelles possibilités de communication et de traitement de l'information, mais mettent aussi en jeu des savoirs qui ne font pas encore partie du fonds culturel commun. Les lignes qui suivent s'intéressent à la question des évolutions curriculaires liées à ces technologies dans l'enseignement obligatoire. Après la présentation de quelques éléments de contexte, destinés à préciser le champ pris en considération, la question des objectifs de formation puis celle des conséquences pour les disciplines de formation existantes seront discutées.

\section{Nouveau contexte}

Des sociétés de plus en plus

« informatisées »

On assiste actuellement, du moins dans les pays industrialisés, à une diffusion générale de ce qu'il est convenu d'appeler les technologies de l'information et de la communication (TIC). Cette diffusion, qui met au cœur de l'actualité des réalités émergentes comme internet et le multimédia, peut être comparée au déferlement d'une "nouvelle vague technologique ", porteuse d'espoirs de changement. D’autres vagues l'ont précédée depuis les années cinquante, chacune tendant à démoder la précédente : après l'audiovisuel, qui a donné lieu à des politiques publiques ambitieuses dans les années soixante, l'intérêt s'est porté, dans les années soixante-dix et quatre-vingt, sur l'informatique, sur la télématique...

En fait, ce qui se développe rapidement, ce sont les dispositifs liés à l'informatique et à l'ordinateur. Depuis la commercialisation des premiers microordinateurs voici une vingtaine d'années, les performances de ces dispositifs n'ont cessé de s'améliorer tandis que leurs coûts baissaient continûment. Les perspectives de développement, telles que les rapportent les médias, semblent sans limite et ce qui aurait été considéré comme de la science-fiction audacieuse voici encore une génération devient crédible ou est déjà réalisé (la reconnaissance vocale, un ordinateur intégré à une montre, un vêtement implanté dans le corps humain pour pallier certains handicaps...). 


\section{De nouveaux instruments}

L'ordinateur n'est rien sans un système logiciel qui lui donne la capacité de communiquer avec des humains. Ces logiciels constituent de nouveaux instruments spécialisés, sans tradition d'usage, permettant de changer profondément les activités et les gestes professionnels. Les plus connus sont les outils de bureautique, dont les premières versions sont apparues au début des années quatre-vingt, et qui sont maintenant relativement banalisés, au moins pour ce qui concerne le traitement de textes. Ils permettent désormais d'incorporer dans des textes des images numériques fixes ou animées et donc de produire des documents ayant un caractère multimédia.

Mais il en existe de bien d'autres types, le spectre allant des systèmes permettant d'accéder à de l'information distante jusqu'aux multimédias spécifiquement destinés à accompagner ou à guider des apprentissages (supports d'un marché parascolaire en pleine expansion), en passant par les jeux encore dits vidéo et les produits du secteur culturel (encyclopédies, musées virtuels...).

La plupart des produits vendus connaissent une évolution rapide, avec des mises à jour fréquentes et partiellement compatibles entre elles. Mais certaines des fonctions qu'ils implémentent (comme le « couper-coller ») tendent à devenir des sortes de classiques.

Même s'ils disposent d'interfaces avec l'usager reposant sur des métaphores graphiques réputées conviviales, les logiciels sont des systèmes techniques complexes et opaques, opérant sur des objets qui ne sont pas ceux dont l'usager a conscience. Leur fonctionnement dépend de paramètres et de principes qui sont loin d'être tous connus.

L'observation montre que les jeunes sont capables, quand ils ont accès à ces instruments, d'en acquérir certaines représentations opératoires. Ces dernières sont néanmoins souvent contextualisées, parfois naïves ou erronées. Elles mettent l'accent sur les objets manipulés (souris, clavier, écran, lecteur de cédérom, icônes de l'interface) et tendent à méconnaître la dimension proprement procédurale du fonctionnement. Les usages sont alors peu résistants aux aléas ou aux changements de version.

Il convient de remarquer, finalement, que les ordinateurs sont encore une ressource incomplètement socialisée et inégalement répartie : même dans un pays industrialisé comme la France, le taux de possession d'ordinateur par foyer était de $20 \%$ en 1998, avec de fortes disparités selon les milieux sociaux ${ }^{1}$. Parmi les jeunes, $50 \%$ avaient, en 1997, accès à un ordinateur à domicile, $70 \%$ dans les milieux sociaux favorisés, $40 \%$ pour les milieux défavorisés ${ }^{2}$. De ce point de vue,

1 Sylvie Dumartin et Frédérique Mignard, "L'informatique à la maison : une diffusion sensible mais encore très ciblée ", INSEE Première, 1999/01, n629.

2 Josiane Jouet et Dominique Pasquier, «Les jeunes et la culture de l'écran ; enquête nationale auprès des 6-17 ans ", CNET-HERMES science publications, Réseaux, 1999, n92-93, pp. 25-102. 
les futurs enseignants ne semblent pas être en retard, puisque nos propres enquêtes montrent que $75 \%$ d'entre eux environ avaient un équipement en entrant, en 1998, en formation initiale.

L'enjeu est que, dans un monde ouvert à la logique de marché, tous les jeunes puissent s'approprier plus que de simples schèmes d'usage de dispositifs informatisés et parviennent à se forger des représentations robustes du traitement automatique de l'information. L'école, au moins pour ce qui concerne l'enseignement obligatoire, est donc concernée au premier chef.

\section{Des conditions nécessaires à l'intégration éducative des technologies}

L'idée qu'il est nécessaire de prendre en compte les nouvelles technologies dans l'éducation est aussi ancienne que leur développement. Au niveau international, l'UNESCO a joué un rôle important dans cette prise de conscience, par l'organisation de colloques internationaux et par la publication de rapports ${ }^{3}$.

Les observations conduites dans les pays industrialisés ont montré puis confirmé un double phénomène. Les phases de recherche et d'innovation pédagogiques menées lors des premières mises à l'épreuve de technologies émergentes ont révélé des potentialités importantes de renouvellement des disciplines enseignées et des activités d'apprentissage. Cependant, leur intégration dans le fonctionnement pédagogique quotidien ne va pas du tout de soi et dépend d'un faisceau de conditions préalables, qui doivent être impérativement satisfaites avant que la définition d'objectifs de formation pour les élèves puisse recevoir une réponse cohérente.

D'abord, comment imaginer que des changements de curriculum dans l'enseignement obligatoire pourraient s'appuyer sur des dispositifs inégalement partagés ? La première condition concerne bien sûr la disponibilité d'équipements (matériel et logiciels). Une fois achetés, ces derniers deviennent assez rapidement obsolètes et doivent être périodiquement remplacés pour faire fonctionner les nouvelles versions des logiciels, ce qui nécessite des investissements dans la durée.

Ensuite, l'usage effectif en classe des TIC suppose que les enseignants (non pas individuellement mais en tant que profession) se les soient appropriées, en aient une maîtrise notable et soient convaincus de leur utilité, ce qui est encore loin d'être le cas ${ }^{4}$. La formation des enseignants, entreprise de longue haleine,

3 Georges-Louis Baron, L'informatique en éducation, quelles évolutions ?, Bulletin du Bureau international d'éducation, $63 \mathrm{e}$ année, $1989, \mathrm{n}^{\circ} 250,96 \mathrm{p}$. ; Éducation et informatique, vers un renforcement de la coopération internationale, Actes du colloque UNESCO, Paris, 12-21 avril 1989, UNESCO, 1990, 2 vol.

4 Georges Baron, Louis Bruillard, L'informatique et ses usagers dans l'éducation, L'éducateur, PUF, Paris, 1996, 312 p. 
joue donc un rôle considérable. Les compétences à développer ne sont pas uniquement techniques; elles concernent également la mise en place et la gestion de nouvelles modalités d'apprentissage dans le contexte des disciplines existantes.

L'utilisation intégrée des technologies demande enfin des changements dans l'organisation pédagogique des écoles et pose de manière insistante la question des infrastructures d'appui aux usagers; jusqu'à présent, en règle générale, seuls les établissements techniques possèdent une " technostructure " suffisante.

Ainsi, les conditions nécessaires à l'intégration des TIC dans l'enseignement obligatoire sont loin d'être partout satisfaites. Cela explique que les évolutions de curriculum qui leur sont liées peuvent se produire surtout dans certains secteurs du système éducatif (et tout particulièrement dans les domaines de l'enseignement technique et professionnel) ou dans certains réseaux scolaires seulement (par exemple, des réseaux privés). Ces évolutions sont de toute façon lentes.

\section{Les objectifs de formation}

Dans les années quatre-vingt, on a vu à l'école élémentaire le lancement expérimental d'enseignements visant des objectifs de culture technique et cherchant à développer chez l'enfant une pensée "logistique ". Le langage de programmation LOGO a servi de base à une approche fondée sur la construction par les élèves de procédures permettant de faire résoudre des problèmes par l'ordinateur. Au collège, l'informatique est entrée dans les enseignements de technologie, sous la forme d'outils de traitement de l'information (particulièrement les traitements de textes) servant à mener des projets de conception et de fabrication.

À l'heure actuelle, l'attention est focalisée sur les nouveaux savoirs que l'école devrait transmettre relativement à l'usage du multimédia et des technologies de la communication. Un consensus est assez facilement atteint sur de grands objectifs généraux de familiarité avec certaines classes d'outils, permettant une adaptation à leur évolution. Mais il est plus difficile de spécifier des objectifs précis, opérationnels, ayant une durée de pertinence suffisante : les fonctionnalités des équipements évoluent, les pratiques sociotechniques de référence sont variables et requièrent des compétences assez différentes en fonction des contextes. Comment prévoir avec certitude des tendances à moyen terme?

Il semble néanmoins qu'on puisse repérer un accord autour d'un ensemble minimal de notions liées aux procédures de gestion de l'immatériel : savoir produire des documents correctement structurés, gérer des fichiers, utiliser de manière courante un tableur, un système de bases de données, un logiciel de dessin vectoriel; savoir rechercher et sélectionner de l'information sur la toile en utilisant un navigateur et en rapatriant une partie de l'information dans d'autres environnements... 
Les objectifs de formation ne sont alors pas relatifs à des produits particuliers, mais au développement de méthodes et de compétences liées à des activités instrumentées. Dès lors, différentes disciplines instituées sont concernées par la question des technologies de l'information et de la communication.

\section{Quelles implications pour les disciplines d'enseignement?}

Dans presque toutes les disciplines, des instruments informatisés sont de nature à renouveler l'approche des phénomènes étudiés, des textes, des images, des documents... Cependant, ils reposent le plus souvent sur des modèles qui sont en décalage par rapport aux contenus traditionnels d'enseignement. Ils permettent de traiter des problèmes complexes, transversaux aux disciplines, de mener avec les élèves des projets mobilisant des savoirs liés à plusieurs d'entre elles. Du coup, ils sont en porte à faux dans un environnement où les temps sont toujours très contraints. La situation est néanmoins différente selon les différents niveaux scolaires.

Dans l'enseignement élémentaire, il existe un véritable foisonnement d'initiatives, surtout quand les écoles ont la capacité de mettre en place des curricula locaux. Ce foisonnement est cependant limité par deux facteurs. Le premier, matériel, tient à la disponibilité d'équipements et aux possibilités de connexion au réseau internet : les écoles du secteur public n’ont pas toujours les moyens d'assurer, seules, les coûts correspondants. Elles doivent alors entrer en partenariat avec d'autres acteurs ou opérateurs, selon des formules relativement hétérogènes. Le second est lié à la formation des enseignants ; comme il a déjà été mentionné ci-dessus, les investissements nécessaires sont importants et ne produisent pas immédiatement leurs effets.

L'enseignement obligatoire de second degré présente un tableau contrasté. Les disciplines de formation générale évoluent lentement et la synchronisation entre elles est pour le moins incomplète. Les traditions culturelles de travail avec des instruments y sont très différentes (ainsi, les disciplines expérimentales ont-elles des traditions de travaux pratiques qui facilitent la réalisation d'expérimentations assistées par ordinateur). Les tentatives d'intégrer les technologies butent souvent sur des problèmes d'éparpillement ou de redondance quand il s'agit de développer chez les élèves des compétences transversales comme le traitement d'images ou la recherche d'information.

Le cas des enseignements à caractère technologique est relativement plus simple, dans la mesure où le recours à des outils et à des instruments y est déjà intégré. Il y est plus facile de modifier les programmes pour les prendre en compte. Une fois installés de nouveaux objectifs de formation et définies des compétences exigibles, le système peut évoluer assez rapidement, au prix néanmoins d'une formation continue des enseignants. 
Cette orientation vers des curricula de technologie de l'information et de la communication, où l'attention des élèves est attirée sur les traitements applicables à cette nouvelle matière d'œuvre, où ils conduisent des activités pratiques finalisées, est déjà adoptée dans différents pays. Elle a le mérite d'armer les élèves de l'enseignement obligatoire de compétences qu'ils pourront sans doute utiliser dans d'autres contextes. 